\title{
Maximin Optimal Designs for a Compartmental Model
}

\author{
Stefanie Biedermann ${ }^{1}$, Holger Dette $^{2}$, and Andrey Pepelyshev ${ }^{3}$ \\ 1 Ruhr-Universität Bochum, Fakultät für Mathematik, 44780 Bochum, Germany. \\ stefanie.biedermann@ruhr-uni-bochum.de \\ 2 Ruhr-Universität Bochum, Fakultät für Mathematik, 44780 Bochum, Germany. \\ holger.dette@ruhr-uni-bochum.de \\ 3 St. Petersburg State University, Department of Mathematics, St. Petersburg, \\ Russia. andrey@ap7236.spb.edu
}

Summary. For an important example from the class of compartmental models we determine optimal designs, which are robust against misspecifications of the unknown model parameters. We propose a maximin approach based on $D$-efficiencies and provide designs that are optimal with respect to the particular choice of various parameter regions.

Key words: Compartmental model, robust optimal design, maximin $D$ optimality, local optimality

\section{Introduction}

We consider a compartmental model, which is commonly applied in the chemical sciences and pharmacokinetics as far as two-step reactions are concerned (see, e.g., [BL59], [GP82] or [At03]). Suppose a substance $A$ decomposes to form substance $B$ which then in turn decomposes to form substance $C$. Assuming that the reactions are irreversible and first order, the concentrations $[A],[B]$ and $[C]$ of products $A, B$ and $C$, respectively, as functions of the time $x \geq 0$ satisfy the kinetic differential equations

$$
\frac{d[A]}{d x}=-\theta_{1}[A], \quad \frac{d[B]}{d x}=\theta_{1}[A]-\theta_{2}[B], \quad \frac{d[C]}{d x}=\theta_{2}[B],
$$

where $\theta_{1}>\theta_{2}>0$ denote unknown constants measuring the specific rates of the first and second decomposition, respectively. Solving system (1) with initial conditions $[A]=1$ and $[B]=[C]=0$ we obtain the amount of intermediate product $B$ after time $x \geq 0$ has elapsed as

$$
\eta(x, \theta)=\frac{\theta_{1}}{\theta_{1}-\theta_{2}}\left(e^{-\theta_{2} x}-e^{-\theta_{1} x}\right) .
$$


In a statistical setup, we thus model observations $Y$ on $[B]$ at different times $x$ as a regression model $Y=\eta(x, \theta)+\varepsilon$, where the observation errors $\varepsilon$ are independent and from an exponential family with zero expectation and constant variance $\sigma^{2}$. The Fisher information $I(x, \theta)$ for estimating the parameter $\theta=\left(\theta_{1}, \theta_{2}\right)^{T}$ at time $x$ is then given by the expression $I(x, \theta)=f(x, \theta) f^{T}(x, \theta)$, where $f(x, \theta)=\left(f_{1}(x, \theta), f_{2}(x, \theta)\right)^{T}$ is the gradient of the regression function $\eta(x, \theta)$ with respect to $\theta$. An (approximate) design $\xi$ is a probability measure with finite support on the non-negative real axis $\mathbb{R}_{0}^{+}$, i.e. the observations are taken at the support points of the measure proportional to the corresponding masses. The Fisher information matrix of the design $\xi$ is given by

$$
M(\xi, \theta)=\int_{0}^{\infty} I(x, \theta) d \xi(x),
$$

and an optimal design maximizes a real-valued function of the Fisher information matrix, which is usually referred to as an optimality criterion (see, e.g., [Sil80]). A typical example is $D$-optimality where the determinant of the Fisher information is maximized with respect to the design $\xi$.

Since an appropriate choice of the experimental conditions can improve the quality of the statistical inference substantially, much effort has been devoted to the problem of finding optimal designs for the compartmental model (2) (see, e.g., [At03], [AC93], [BL59], [DO99], [HW85] or [HH74]). The Fisher information and thus the optimal designs with respect to the common optimality criteria depend on the unknown parameter. Following [Ch53], several authors assume that an initial guess of $\theta$ is available and determine so-called locally optimal designs (see [BL59] or [HH74]). However, misspecifications of the parameter for a locally optimal design can lead to poor results in the subsequent data analysis. A more robust alternative is to assume sufficient knowledge of $\theta$ to specify a prior distribution for this parameter and to average the respective optimality criteria over the plausible values of $\theta$ defined by the prior. This leads to so-called Bayesian optimality criteria (see, e.g., [AC93] or [PW85]). As an alternative for the construction of robust designs, we propose a maximin approach based on $D$-efficiencies, which only requires the specification of a certain range for the unknown parameter. We feel that this is a more realistic scenario since practitioners will often have difficulties to specify a prior distribution for the unknown parameter $\theta$. Most of the literature on maximin design discusses models with only one nonlinear parameter or minimally supported designs (see,e.g., [Im01]). Model (2), however, contains two nonlinear parameters and therefore standard techniques are not readily applicable. In section 2, we provide some insight in the structure of locally $D$-optimal designs for the compartmental model (2). In section 3, we define the standardized maximin $D$-optimality criterion, determine various designs optimal with respect to the maximin criterion and investigate the properties of these optimal designs. It turns out that in many cases the standardized maximin $D$-optimal designs are supported on three points so that they can also be used for model checking. 


\section{Locally $D$-optimal designs}

We commence our investigation of the locally $D$-optimal designs by presenting a result relating $D$-optimal designs with respect to several parameter combinations, thus yielding a substantial simplification of the optimization problem.

Lemma 1. Let $x^{*}(\theta)$ denote the vector of support points of the locally Doptimal design with respect to the parameter $\theta$. Then

$$
x^{*}(\gamma \theta)=\frac{1}{\gamma} x^{*}(\theta)
$$

for any $\gamma>0$. Moreover, the locally D-optimal designs with respect to the parameters $\theta$ and $\gamma \theta$ have the same weights at the corresponding support points.

Proof. The assertion of Lemma 1 is a simple consequence of the homogeneity of the linear differential equation formulation (1) and applies quite generally.

By Lemma 1 it is sufficient to compute locally $D$-optimal designs with $\theta_{1}=1$, since the optimal designs with respect to any other parameter can easily be obtained from these designs by rescaling.

Numerical calculations suggest that the locally $D$-optimal designs in the compartmental model (2) are supported on exactly two points. Furthermore, minimally supported $D$-optimal designs are equally weighted, which is a standard result in design theory (see, e.g., [Sil80], Lemma 5.1.3). Some locally Doptimal designs for several representative situations with respect to the choice of $\theta$ are listed in Table 1.

Table 1. The support points $x_{1}^{*}, x_{2}^{*}$ of the locally $D$-optimal designs $\xi^{*}=$ $\left\{x_{1}^{*}, x_{2}^{*} ; 1 / 2,1 / 2\right\}$ with respect to the parameter $\theta=\left(1, \theta_{2}\right)^{T}$.

\begin{tabular}{lllllllll}
\hline$\theta_{2}$ & $x_{1}^{*}$ & $x_{2}^{*}$ & $\theta_{2}$ & $x_{1}^{*}$ & $x_{2}^{*}$ & $\theta_{2}$ & $x_{1}^{*}$ & $x_{2}^{*}$ \\
\hline 0.1 & 0.9283 & 11.0171 & 0.4 & 0.8186 & 3.9018 & 0.7 & 0.7164 & 2.8599 \\
0.2 & 0.8907 & 6.1603 & 0.5 & 0.7825 & 3.4353 & 0.8 & 0.6868 & 2.6634 \\
0.3 & 0.8554 & 4.6515 & 0.6 & 0.7483 & 3.1076 & 0.9 & 0.6594 & 2.5020 \\
\hline
\end{tabular}

As an interesting result from Table 1 we obtain that a large distance between $\theta_{1}$ and $\theta_{2}$ results in relatively large support points of the locally $D$ optimal designs $\xi^{*}$. In the following lemma, we show a result on the asymptotic behavior of the design $\xi^{*}$ when $\theta_{2}$ tends to its upper boundary.

Lemma 2. If $\theta_{2}$ tends to $1\left(=\theta_{1}\right)$ from below, the locally D-optimal design $\xi^{*}=\xi^{*}\left(\theta_{2}\right)$ converges weakly to the design with support points $x_{1}^{*}=(3-\sqrt{3}) / 2$, $x_{2}^{*}=(3+\sqrt{3}) / 2$ and equal weights, which is $D$-optimal for the linear regression model

$$
Y=a_{1}\left(x-\frac{x^{2}}{2}\right) e^{-x}+a_{2} \frac{x^{2}}{2} e^{-x}+\varepsilon .
$$


Proof. Let $\theta_{2}=1-z$. By a Taylor expansion of the regression gradient at the point $z=0$ we obtain

$$
f_{1}(x, \theta)=\left(x-\frac{x^{2}}{2}\right) e^{-x}+o(z), f_{2}(x, \theta)=-\frac{x^{2}}{2} e^{-x}+o(z),
$$

and the assertion follows.

So far, we have considered design regions for the time $x$, which are unbounded, i.e. the time $x$ is allowed to vary from zero to infinity. However, in many situations there exists a boundary, $x_{\max }$ say, on the maximal amount of time that is feasible for the particular experiment. In this case, Lemma 1 has to be modified appropriately, i.e. if $\xi^{*}=\left(x_{i}^{*} ; w_{i}^{*}\right)$ is the locally $D$-optimal design with respect to the parameter $\theta$ on the design space $\left[0, x_{\text {max }}\right]$ with support points $x_{i}^{*}$ and corresponding weights $w_{i}^{*}$, then $\xi_{\gamma}^{*}=\left(\frac{1}{\gamma} x_{i}^{*} ; w_{i}^{*}\right)$ is locally $D$-optimal with respect to the parameter $\gamma \theta$ on the design space $\left[0, \frac{1}{\gamma} x_{\max }\right]$. Table 2 gives several examples of locally $D$-optimal designs for the compartmental model (2) with a restricted design space. The efficiencies (eff) of the restricted designs $\xi_{x_{\max }}^{*}$ are given by the square root of the ratio of their particular criterion value and the criterion value of the locally $D$-optimal design $\xi^{*}$ with respect to an unrestricted design interval.

Table 2. Locally $D$-optimal designs with respect to several restricted design spaces $\left[0, x_{\max }\right]$ and different values of $\theta$.

\begin{tabular}{|c|c|c|c|c|c|c|c|c|c|c|c|}
\hline \multicolumn{4}{|c|}{$\theta=(1,0.5)^{T}$} & \multicolumn{4}{|c|}{$\theta=(1,0.1)^{T}$} & \multicolumn{4}{|c|}{$\theta=(1,0.05)^{T}$} \\
\hline$x_{\max }$ & $x_{1}^{*}$ & $x_{2}^{*}$ & eff & $x_{\max }$ & $x_{1}^{*}$ & $x_{2}^{*}$ & eff & $x_{\max }$ & $x_{1}^{*}$ & $x_{2}^{*}$ & eff \\
\hline$\infty$ & 0.783 & 3.435 & 1 & $\infty$ & 0.928 & 11.017 & 1 & $\infty$ & 0.95 & 21.004 & 1 \\
\hline 3 & 0.758 & & 0.979 & 10 & 0.928 & 10 & 0.994 & 19 & 0.95 & 819 & 0.995 \\
\hline 2.5 & 0.713 & 2.5 & 0.891 & 8 & 0.932 & 8 & 0.944 & 15 & 0.95 & 815 & 0.945 \\
\hline 2 & 0.646 & & 0.728 & 6 & 0.936 & & 0.820 & 11 & 0.95 & 811 & 0.824 \\
\hline 1.5 & 0.548 & 1.5 & 0.495 & 4 & 0.914 & & 0.589 & 7 & 0.96 & 37 & 0.603 \\
\hline 1 & 0.410 & & 0.240 & 2 & 0.717 & & 0.224 & 3 & 0.87 & 53 & 0.234 \\
\hline 0.5 & 0.228 & 0.5 & 0.049 & 1 & 0.436 & & 0.053 & 1 & 0.44 & 11 & 0.027 \\
\hline 0.25 & 0.120 & 0.25 & 0.008 & 0.5 & 0.236 & 0.5 & 0.009 & 0.5 & 0.23 & 60.5 & 0.005 \\
\hline
\end{tabular}

Observing the entries of Table 2, we notice that if the largest support point of the unrestricted $D$-optimal design $\xi^{*}$ is larger than $x_{\max }$ the boundary itself is in the support of $\xi_{x_{\max }}^{*}$. Moreover, the efficiencies of the restricted optimal designs decrease considerably with decreasing $x_{\max }$. As a consequence, we suggest using the unrestricted design whenever possible, else trying to choose the boundary $x_{\max }$ as large as possible from the experimental circumstances. 


\section{Standardized maximin $D$-optimal designs}

Following [Mü95] or [Im01] we propose as robust designs for the compartmental model (2) designs, which maximize the minimal $D$-efficiency calculated over a certain range for the parameter $\theta$, thus protecting the experiment against the worst case scenario. That means that we maximize the expression

$$
\Psi_{-\infty}(\xi)=\inf _{\theta \in \Theta}\left[\left(\frac{\operatorname{det} M(\xi, \theta)}{\operatorname{det} M\left(\xi_{\theta}^{*}, \theta\right)}\right)^{1 / 2}\right],
$$

where, throughout this article, $\xi_{\theta}^{*}$ denotes the locally $D$-optimal design with respect to $\theta$. A design maximizing $\Psi_{-\infty}(\xi)$ is called standardized maximin $D$-optimal (with respect to $\Theta$ ). The parameter space $\Theta$ of possible values of $\theta$ has to be specified in advance by the experimenter. An advantage of this approach compared to a Bayesian setup is that it is not required to specify a prior distribution for the unknown parameter $\theta$, which is not possible in all circumstances. The only "prior knowledge" needed to use the standardized maximin $D$-criterion is an approximate range $\Theta$ for the parameter $\theta$.

A powerful tool for checking maximin optimality of a design is an equivalence theorem, which can be found in [DH03].

Theorem 1. A design $\xi^{*}$ is standardized maximin D-optimal with respect to $\Theta$ if and only if there exists a prior $\pi^{*}$ supported on the set

$$
\mathcal{N}\left(\xi^{*}\right)=\left\{\theta \in \Theta \mid \Psi_{-\infty}\left(\xi^{*}\right)=\left(\frac{\operatorname{det} M(\xi, \theta)}{\operatorname{det} M\left(\xi_{\theta}^{*}, \theta\right)}\right)^{1 / 2}\right\}
$$

such that the inequality

$$
d\left(\xi^{*}, x\right)=\int_{\mathcal{N}\left(\xi^{*}\right)} f^{T}(x, \theta) M^{-1}\left(\xi^{*}, \theta\right) f(x, \theta) d \pi^{*}(\theta) \leq 2
$$

holds for all $x$ within the design space. Moreover, there is equality in (5) for all support points of the design $\xi^{*}$.

Following [DH03] we call the prior $\pi^{*}$ least favourable distribution. The designs given in the following discussion were calculated numerically using Theorem 1 whereby their optimality was carefully checked. These calculations are quite complicated, since the least favourable distribution is twodimensional. A MATLAB-programme is available from the third author.

There are two obvious ways, in which a parameter space $\Theta$ can be specified. On the one hand, it might be sensible to assume a one-dimensional closed interval as an admissible range for each parameter. Since the inequality $\theta_{1}>$ $\theta_{2}>0$ always holds, that means that $\Theta$ is given by a closed rectangle in the positive half plane, i.e. $\Theta=\Theta\left(z_{1}, z_{2}, z_{3}, z_{4}\right):=\left[z_{1}, z_{2}\right] \times\left[z_{3}, z_{4}\right], z_{i}>0, z_{1}>$ $z_{4}$. On the other hand, the fact that $\theta_{1}>\theta_{2}$ can result in a triangular region $\Theta$, i.e. $\Theta=\Theta\left(z_{1}, z_{2}\right):=\left\{\left(z_{1}, z_{2}\right) \mid 0<z_{1} \leq \theta_{2}<\theta_{1} \leq z_{2}\right\}$.

The following lemma applies Lemma 1 to simplify the maximin optimization problem for $\Theta$ from the above-mentioned cases considerably. 
Lemma 3. Denote by $x_{i}^{*}=x_{i}^{*}\left(z_{1}, \ldots, z_{j}\right)$ the support points of the standardized maximin $D$-optimal design with respect to $\Theta\left(z_{1}, \ldots, z_{j}\right), j=4$ or $j=2$. For any $\gamma>0$ the design with the same weights and support points $\frac{1}{\gamma} x_{i}^{*}$ is $\Psi_{-\infty}$-optimal with respect to the parameter space $\gamma \Theta=\Theta\left(\gamma z_{1}, \ldots, \gamma z_{j}\right)$.

If $x_{i}$ denote the support points of the $\Psi_{-\infty}$-optimal design with respect to $\Theta$ on the restricted design space $\left[0, x_{\max }\right]$, then the design with the same weights and support points $\frac{1}{\gamma} x_{i}$ is $\Psi_{-\infty}$-optimal with respect to $\gamma \Theta$ on $\left[0, \frac{1}{\gamma} x_{\max }\right]$.

Proof. The standardized maximin $D$-optimal design with respect to $\gamma \Theta$ can be obtained by maximizing

$$
\begin{aligned}
& \min _{\theta \in \gamma \Theta} \frac{\operatorname{det} \int_{0}^{x_{\max }} I(x, \theta) d \xi(x)}{\operatorname{det} \int_{0}^{x_{\max }} I(x, \theta) d \xi_{\theta}^{*}(x)}=\min _{\theta \in \Theta} \frac{\operatorname{det} \int_{0}^{x_{\max }} I(x, \gamma \theta) d \xi(x)}{\operatorname{det} \int_{0}^{x_{\max }} I(x, \gamma \theta) d \xi_{\theta}^{*}(x)} \\
= & \min _{\theta \in \Theta} \frac{\frac{1}{\gamma^{4}} \operatorname{det} \int_{0}^{x_{\max }} I(\gamma x, \theta) d \xi(x)}{\frac{1}{\gamma^{4}} \operatorname{det} \int_{0}^{x_{\max }} I(\gamma x, \theta) d \xi_{\theta}^{*}(x)}=\min _{\theta \in \Theta} \frac{\operatorname{det} \int_{0}^{\frac{x_{\max }}{\gamma}} I(x, \theta) d \tilde{\xi}(x)}{\operatorname{det} \int_{0}^{\frac{x_{\max }}{\gamma}} I(x, \theta) d \xi_{\gamma \theta}^{*}(x)},
\end{aligned}
$$

where the design $\tilde{\xi}$ is derived from $\xi$ by the relation $\tilde{\xi}(\{x\})=\xi(\{\gamma x\})$. The above equalities are obtained by direct calculations on the entries of $I(x, \theta)$, whereas the last transformation in the denominator is an application of Lemma 1.

In Tables 3 and 4 we present some $\Psi_{-\infty}$-optimal designs corresponding to rectangular parameter regions. The term min $\mathrm{eff}_{D}$ stands for the criterion value of the optimal design. The intervals $\left[z_{1}, z_{2}\right],\left[z_{3}, z_{4}\right]$ in Table 3 are chosen relat-

Table 3. Standardized maximin $D$-optimal designs $\xi^{*}$ for model (2) with unrestricted design space with respect to various rectangular parameter spaces.

\begin{tabular}{llllllllllllll}
\hline$z_{1}$ & $z_{2}$ & $z_{3}$ & $z_{4}$ & $x_{1}$ & $x_{2}$ & $\min \mathrm{eff}_{D}$ & $z_{1}$ & $z_{2}$ & $z_{3}$ & $z_{4}$ & $x_{1}$ & $x_{2}$ & $\operatorname{min~} \mathrm{eff}_{D}$ \\
\hline 0.7 & 0.8 & 0.3 & 0.4 & 1.06 & 4.78 & 0.983 & 0.9 & 1.1 & 0.2 & 0.5 & 0.86 & 4.40 & 0.891 \\
0.9 & 1.0 & 0.3 & 0.4 & 0.88 & 4.33 & 0.986 & 2.2 & 2.8 & 0.2 & 0.5 & 0.37 & 3.47 & 0.885 \\
0.9 & 1.1 & 0.3 & 0.4 & 0.84 & 4.25 & 0.978 & 2.2 & 2.8 & 0.2 & 0.7 & 0.37 & 2.94 & 0.809 \\
0.9 & 1.1 & 0.3 & 0.5 & 0.83 & 3.95 & 0.954 & 2.0 & 3.0 & 0.2 & 0.7 & 0.37 & 2.93 & 0.787 \\
\hline
\end{tabular}

ively small. In this case, the $\Psi_{-\infty}$-optimal designs are supported on two points and equally weighted. For the examples in Table 4 we choose larger parameter regions $\Theta$, thus obtaining optimal designs supported on three points. Results for triangular parameter regions are depicted in Table 5 .

In our numerical study, we observe that standardized maximin $D$-optimal designs with respect to rectangular or triangular parameter spaces have at most three support points. A third support point appears if a "large" $\Theta$ is chosen, since these $\Psi_{-\infty}$-optimal designs must be "good" despite a high level of uncertainty about the position of the parameters. In some cases a relatively 
Table 4. Standardized maximin $D$-optimal designs $\xi^{*}$ for model (2) with unrestricted design space with respect to various rectangular parameter spaces.

\begin{tabular}{lllllllllll}
\hline$z_{1}$ & $z_{2}$ & $z_{3}$ & $z_{4}$ & $x_{1}$ & $x_{2}$ & $x_{3}$ & $w_{1}$ & $w_{2}$ & $w_{3}$ & $\min \mathrm{eff}_{D}$ \\
\hline 2 & 3 & 0.2 & 0.8 & 0.37 & 2.28 & 4.69 & 0.50 & 0.35 & 0.15 & 0.755 \\
2 & 3 & 0.2 & 0.9 & 0.35 & 1.97 & 4.80 & 0.49 & 0.32 & 0.19 & 0.736 \\
2 & 3 & 0.2 & 1 & 0.35 & 1.63 & 5.14 & 0.50 & 0.29 & 0.21 & 0.727 \\
2 & 3 & 0.1 & 1 & 0.38 & 1.85 & 7.88 & 0.54 & 0.25 & 0.20 & 0.661 \\
\hline
\end{tabular}

Table 5. Standardized maximin $D$-optimal designs for model (2) with unrestricted design space with respect to various triangular parameter regions.

\begin{tabular}{lllllllll}
\hline$z_{1}$ & $z_{2}$ & $x_{1}$ & $x_{2}$ & $x_{3}$ & $w_{1}$ & $w_{2}$ & $w_{3}$ & $\min \mathrm{eff}_{D}$ \\
\hline 1 & 2 & 0.44 & 1.64 & & 0.50 & 0.50 & & 0.822 \\
0.5 & 1 & 0.88 & 3.28 & & 0.50 & 0.50 & & 0.822 \\
0.4 & 1 & 0.79 & 2.43 & 5.76 & 0.38 & 0.39 & 0.22 & 0.761 \\
1 & 3 & 0.25 & 0.84 & 2.18 & 0.35 & 0.41 & 0.24 & 0.740 \\
0.3 & 1 & 0.83 & 2.61 & 7.15 & 0.36 & 0.41 & 0.22 & 0.728 \\
\hline
\end{tabular}

small number of different stages for the experiment can reduce costs considerably. Moreover, three design points still allow model checking to some extent.

We, finally, deal with the problem of finding $\Psi_{-\infty}$-optimal designs on a restricted design space. The results which are similar for rectangular and triangular parameter regions $\Theta$ are given in Tables 6 and 7. Unlike our obser-

Table 6. Standardized maximin $D$-optimal designs for model (2) with respect to various rectangular parameter regions on restricted design spaces.

\begin{tabular}{lllllllllllll}
\hline$\Theta=[0.8,1.2] \times[0.2,0.5]$ & \multicolumn{1}{c}{$\Theta=[2,3] \times[0.2,1]$} \\
\hline$x_{\max }$ & $x_{1}$ & $x_{2}$ & $\operatorname{min~} \mathrm{eff}_{D}$ & $x_{\max }$ & $x_{1}$ & $x_{2}$ & $x_{3}$ & $w_{1}$ & $w_{2}$ & $w_{3}$ & $\min \mathrm{eff}_{D}$ \\
\hline$\infty$ & 0.86 & 4.41 & 0.854 & $\infty$ & 0.36 & 1.64 & 5.29 & 0.50 & 0.29 & 0.21 & 0.727 \\
4 & 0.79 & 3.89 & 0.943 & 4 & 0.35 & 1.69 & 4 & 0.51 & 0.28 & 0.21 & 0.741 \\
3 & 0.79 & 3 & 0.984 & 3 & 0.35 & 1.85 & 3 & 0.49 & 0.31 & 0.20 & 0.785 \\
2 & 0.67 & 2 & 0.992 & 2 & 0.33 & 1.91 & 0.5 & 0.5 & 0.909 \\
1 & 0.42 & 1 & 0.998 & 1 & 0.31 & 1 & & 0.5 & 0.5 & 0.986 \\
0.5 & 0.23 & 0.5 & 1.000 & 0.5 & 0.20 & 0.5 & & 0.5 & 0.5 & & 0.996 \\
\hline
\end{tabular}

vations concerning locally $D$-optimal designs, we find that the largest support point of the restricted maximin optimal designs is not always given by the boundary value. Another noticeable aspect is that in both the rectangular and the triangular case the number of support points of the restricted design decreases with decreasing the boundary, whereas the $D$-efficiencies increase. 
Table 7. Standardized maximin $D$-optimal designs for model (2) with respect to various triangular parameter regions on restricted design spaces.

\begin{tabular}{lllllllllllll}
\hline \multicolumn{1}{c}{$z_{1}=0.5, z_{2}=1$} & \multicolumn{1}{c}{$z_{1}=0.3, z_{2}=1$} \\
\hline$x_{\max }$ & $x_{1}$ & $x_{2}$ & $\min \mathrm{eff}_{D}$ & $x_{\max }$ & $x_{1}$ & $x_{2}$ & $x_{3}$ & $w_{1}$ & $w_{2}$ & $w_{3}$ & $\min$ eff \\
\hline$\infty$ & 0.88 & 3.28 & 0.82 & $\infty$ & 0.83 & 2.62 & 7.12 & 0.36 & 0.41 & 0.22 & 0.728 \\
3 & 0.78 & 2.91 & 0.93 & 5 & 0.75 & 2.25 & 5 & 0.33 & 0.41 & 0.26 & 0.759 \\
2 & 0.68 & 2 & 0.98 & 3 & 0.81 & 3 & & 0.5 & 0.5 & & 0.904 \\
1 & 0.42 & 1 & 0.99 & 2 & 0.73 & 2 & & 0.5 & 0.5 & 0.963 \\
0.5 & 0.23 & 0.5 & 0.99 & 1 & 0.44 & 1 & & 0.5 & 0.5 & 0.979 \\
\hline
\end{tabular}

Acknowledgements: The support of the Deutsche Forschungsgemeinschaft (SFB 475, Komplexitätsreduktion in multivariaten Datenstrukturen, Teilprojekt A2, Sachbeihilfe De 502/18-1) is gratefully acknowledged.

\section{References}

[At03] Atkinson, A.C.: Horwitz's rule, transforming both sides and design of experiments for mechanistic models. Applied Statistics, 52, 261-278 (2003)

[AC93] Atkinson, A.C., Chaloner, K., Herzberg, A.M., Juritz, J.: Optimum experimental designs for properties of a compartmental model. Biometrics, 49, 325-337 (1993)

[BL59] Box, G.E.P., Lucas, H.: Design of experiments in non-linear situations. Biometrika, 46, 77-90 (1959)

[Ch53] Chernoff, H.: Locally optimal designs for estimating parameters. Annals of Mathematical Statistics, 24, 586-602 (1953)

[DH03] Dette, H., Haines, L. and Imhof, L.: Maximin and Bayesian optimal designs for regression models. (2003) http://www.ruhr-unibochum.de/mathematik3/preprint.htm

[DO99] Dette, H., O’Brien, T.E.: Optimality criteria for regression models based on predicted variance. Biometrika, 86, 93-106 (1999)

[GP82] Gibaldi, M., Perrier, D.: Pharmacokinetics. Second edition. Dekker, New York (1982)

[HW85] Hamilton, D.C., Watts D.G.: A quadratic design criterion for precise estimation in nonlinear regression models. Technometrics, 27, 241-250 (1985)

[HH74] Hill, W.J., Hunter W.G.: Design of experiments for subsets of parameters. Technometrics, 16, 425-434 (1974)

[Im01] Imhof, L.: Maximin designs for exponential growth models and heteroscedastic polynomial models. Annals of Statistics, 29, 561-576 (2001)

[Mü95] Müller, C. H.: Maximin efficient designs for estimating nonlinear aspects in linear models. Journal of Statistical Planning and Inference, 44, 117-132 (1995)

[PW85] Pronzato, L., Walter, E.: Robust experiment design via stochastic approximation. Mathematical Biosciences, 75, 103-120 (1985)

[Sil80] Silvey, S.D.: Optimal Design. Chapman and Hall, London (1980) 